\title{
Lectura hipertextual en un contexto de alfabetización académica: un estudio de caso ${ }^{1}$
}

\section{Hypertextual Reading in a Context of Academic Literacy: A Case Study}

\author{
Mariana Sofía AILLON NEUMANN, Beatriz Eugenia FIGUEROA SANDOVAL \\ y Angie Carolina NEIRA MARTÍNEZ \\ Universidad de Concepción - Chile
}

Recibido: Marzo 2014

Aceptado: Julio 2014

\begin{abstract}
Resumen
Con el acceso y uso masivos de las TIC, la demanda de aplicaciones educativas se ha incrementado. En este escenario, se han conformado equipos interdisciplinarios, y se han destinado tiempo y recursos en la creación de software multimodales y el manejo de hipermedia e internet para enseñar a los estudiantes a leer, escribir y comunicarse en línea. Los resultados no solo demuestran su efectividad en la enseñanza de lenguas, sino también en la alfabetización digital, en los distintos niveles educativos. Este antecedente ha generado el interés de especialistas del ámbito de la alfabetización académica hipertextual, para responder a las necesidades que presentan los estudiantes de enseñanza superior respecto del uso de internet, específicamente en la obtención información y generación de conocimiento. Para plantear soluciones a esta situación, es preciso identificar los procesos cognitivos que desarrollan los sujetos en su interacción con la red. Ante este desafío, el presente artículo recoge los hallazgos de un estudio de caso realizado con el software Netsupport School que permiten describir la práctica de lectura hipertextual de estudiantes de Magíster en Educación. El trabajo ofrece una identificación y análisis de los procesos cognitivos desarrollados durante la realización de una tarea específica de búsqueda, selección y asociación de información en internet, para una asignatura de un Postgrado de Educación. Los resultados indican que el desarrollo de prácticas de hiperlectura es imprescindible al enfrentar las tareas académicas que exigen los estudios de postgrado en la actualidad y que un manejo deficiente de las prácticas de hiperlectura impactan negativamente en los aprendizajes de los estudiantes.
\end{abstract}

Palabras clave: alfabetización académica, lectura, hipertexto, estudio de caso.

1 El presente estudio forma parte de un Proyecto Fondecyt Regular № 1110909 "Alfabetización académica: el hipertexto una herramienta para mejorar los aprendizajes en la formación de profesores", financiado por CONICYT (Comisión Nacional de Investigación Científica y Tecnológica) de Chile. 


\begin{abstract}
Considering the massive access and use of ICTs the demand for educational applications has increased. In this particular scenario interdisciplinary teams have been generated and time and resources have been allocated for the creation of multimodal softwares and the management of the hypermedia and the internet to train students on how to read, write and communicate online. Results not only show their efficiency in language teaching, but also in digital literacy, in the different educational levels. This background has aroused the interest of specialists related to the hypertextual academic literacy environment to meet the needs of higher education students with respect to the use of internet, especially in the procurement of information and generation of knowledge.

To be able to suggest solutions with respect to this particular situation it is necessary to identify on first place the cognitive processes which is developed by the individuals in their interaction with the web. Regarding this challenge, the present study considers the findings of a case study performed with the assistance of the Netsupport School Software that describes the practice of hypertextual reading, carried out by a group of students belonging to a Master's Degree in Education program.

The research work shows the identification and the analysis of cognitive processes developed during the performance of a particular task related to the search of information, selection and association of information in internet for a specific subject matter of a Post-Degree program in Education. Results suggest that the development of hyper-reading practices is essential to be able to face academic duties requested by current post-graduate studies and that an imperfect management of hyper-reading practices will negatively impact on students learning.
\end{abstract}

Keywords: academic literacy, reading, hypertext, case study.

La implementación de los programas de alfabetización enfrenta, en la actualidad, una situación compleja y experimenta profundos cambios debido, fundamentalmente, al acelerado avance de las Tecnologías de la Información y Comunicación (TIC) (Escolano, 2006; Colom, 2002). Este escenario ha llevado a los investigadores de distintas disciplinas a explorar y generar aplicaciones tecnológicas para apoyar las mediaciones didácticas, e indagar sobre los efectos de los nuevos dispositivos móviles en distintos niveles educativos, como un reciente estudio realizado por Sevillano y Vásquez (2014) en tres universidades españolas (Universidad Complutense de Madrid, Universidad de Oviedo y Universidad Nacional de Educación a Distancia), cuyos resultados destacan la contribución de las tabletas digitales en el desarrollo de competencias genéricas instrumentales en la Sociedad del Conocimiento, a saber: la búsqueda y organización de información (Descriptores de Dublín, 2005)

Sin duda, el impacto de internet en los procesos vinculados a la consulta y tratamiento de la información ha modificado las prácticas de lectura y escritura en la universidad, situación que es asumida como un desafío por la alfabetización académica.

El término alfabetización académica tiene su origen en el medio anglosajón (academic literacy) y es definido por una de sus principales investigadoras latinoamericanas como "el conjunto de nociones y estrategias necesarias para participar en la cultura discursiva de las disciplinas así como las actividades de producción y análisis de textos requeridas para aprender en la universidad" (Carlino, 2007: 13). Esta 
definición se refiere no solo a las prácticas de lectura y escritura propias de la Educación Superior y las formas de representar el conocimiento en cada área disciplinaria, sino también a los requisitos para ingresar en la comunidad académica. Esta última dimensión es clave para comprender la complejidad del proceso de formación que implica para el estudiante una carrera universitaria. Esta es una "cultura" ajena para el aprendiz y, por lo tanto, él precisa de herramientas y estrategias para apropiarse del discurso que proyecta el esquema de pensamiento que caracteriza su área de estudio (Carlino, 2007; Parodi, 2010).

Las dificultades que enfrentan los estudiantes son mayores si se considera que la fuente de información y conocimiento primordial para apoyar sus tareas es internet. $\mathrm{Si}$ bien este soporte tecnológico es utilizado constantemente y con experticia en contextos recreativos, en el ámbito de la búsqueda y procesamiento de información académica es manejada en forma incipiente. Esto ocurre, por una parte, debido a la arquitectura hipertextual que obliga a una lectura no secuencial entre los nodos de información, a los cuales se accede a través de los links que van eligiendo los jóvenes, en la mayoría de los casos intuitivamente. Por otra parte, ante una cantidad infinita de material y múltiples caminos de lectura, sin los conocimientos disciplinarios básicos, la consulta en la red puede ser una experiencia frustrante con escasos resultados en el aprendizaje, como lo prueba la investigación doctoral de Albarello (2010) realizada con profesores y jóvenes adolescentes, para estos últimos resultaba tedioso navegar por internet con escaso conocimiento sobre el tema para cumplir con sus tareas. Los dos aspectos mencionados justifican el desarrollo de una alfabetización digital que enfatice la gestión del conocimiento en un campo disciplinario específico, producto del manejo de la tecnología que sirve de soporte a la información disponible.

Lo anterior se fundamenta en la hipótesis que plantea que el hipertexto no solo ha transformado las prácticas de lectura y escritura de los estudiantes universitarios, sino que además estos cambios han influido en su forma de pensar y aprender. Esta última idea ha sido uno de los focos de interés para los investigadores en psicología educacional (Monereo, Fuentes, Sànchez, 2000; Coll y Monereo, 2008), tecnología educativa (Stone, Rennebohm y Breit 2006; Rueda, 2007; Piscitelli, 2009), didáctica de la lengua (Figueroa et al., 2010) y lingüística aplicada (Warschauer, 2000; Mayer y Moreno, 2003). Aún queda bastante camino por recorrer, ya que no se ha llevado a cabo una distinción disciplinaria en la navegación por internet, lo que implica objetivos, tareas, productos y sitios web específicos que presentan características determinadas y, por lo tanto, condicionan la consulta en la red. A los factores anteriores, se agrega la complejidad para acceder y estudiar los procesos cognitivos, y la imprescindible dependencia, en una buena medida, de la explicitación de dichos procesos por parte de los sujetos usuarios durante el desarrollo de sus prácticas lectoescritas. Algunos estudios han recogido, mediante entrevistas, los procesos desde una perspectiva declarativa previa o posterior a ejercicios de hiperlectura (Albarello, 2010) o a través de encuestas sobre hábitos asociados al uso de Internet (Sevillano y Vásquez, 2014).

En el marco anterior, este trabajo tiene como objetivo describir la práctica de lectura hipertextual de un grupo de estudiantes de Magíster en Educación. La idea es 
identificar y analizar los procesos cognitivos desarrollados durante la ejecución de una tarea específica de búsqueda, selección y asociación de información en internet para una asignatura específica del programa de formación. Para ello, se realizó un estudio de caso con la utilización del software Netsupport. La aplicación del software tiene como propósito registrar, en modalidad sincrónica, las acciones de los hiperlectores a medida que llevaban a cabo la tarea encomendada. De esta forma, Netsupport optimiza la recogida de información de una investigación sobre la lectura hipertextual.

Los resultados obtenidos en este estudio de caso aportan datos que se constituyen en insumos valiosos para alcanzar los objetivos de la investigación del Proyecto Fondecyt $\mathrm{N}^{\circ} 1110909$, que se plantea, entre otras cosas: "Describir, analizar y potenciar el uso de herramientas tecnológicas multimodales como artefactos de mediación y transformación del conocimiento desde un diseño didáctico que busca desarrollar la alfabetización hipertextual”.

El desarrollo del artículo se organiza en cuatro apartados. En el primer apartado se exponen los principios teóricos involucrados en la comprensión de los procesos cognitivos que experimentan los sujetos en la navegación por internet; en el segundo, se describe la metodología aplicada en el estudio; en el tercero, se presentan los resultados obtenidos y en el cuarto se dan a conocer las conclusiones.

\section{Principios teóricos de los procesos cognitivos implicados en la lectura hipertextual}

La lectura en internet se puede definir como una actividad interactiva, cognitiva y perceptual de navegación en un soporte hipertextual. Esta lectura no secuencial se desarrolla en forma lateral, que consiste en la selección estratégica de una cantidad de links para acceder a la información requerida (Fainholc, 2004). Este proceso está orientado a la construcción de sentido, es decir, a la interpretación del contenido obtenido de la red. Esto implica necesariamente la resignificación de un material fragmentado, elaborado y/o difundido por otros, que se logra por los vínculos que establece, ya no la máquina, sino el (hiper)lector, para integrar "cada dato y cada relación dentro de un conjunto que otorga su significado, su peso y su valor de uso" (Chartier, 2004: 198).

La lectura hipertextual también potencia otros tipos de lectura: vertical, que elige el internauta; direccional, que responde a los objetivos o interés de información del sujeto; secuenciada, que se realiza de una ventana a otra; simultánea, que se trabaja con dos contenidos relacionados que aparecen en la misma pantalla (Fainholc, 2004).

En esta práctica de lectura on line se desarrollan procesos que actualizarían diferentes procedimientos o destrezas, algunos reflexivos y otros automáticos, para integrar información de distinta naturaleza:

sintáctica (al relacionar signos diversos dentro de un relato); esquemática (o como plan o bosquejo, parcial o filtrada, vinculada a la representación de una figura sin entrar en sus detalles), audiovisual (si considera la imagen fija o móvil y/o la música, ruidos, efectos, etc.); argumentativa (que sirve para 
deducir, inferir, demostrar, etc., supuestos del relato apelando a sus funciones abstractas de pensamiento); semántica (porque focaliza los campos significativos de las lexías o referentes); pragmática (o según la perspectiva de la aplicación que de los textos realice el usuario); léxica, gráfica e interpretativa, etc. (Fainholc, 2004: 33)

La complejidad de los procesos involucrados en la lectura hipertextual precisa de la activación de los conocimientos previos del individuo sobre el contenido de la búsqueda, los objetivos que guían la hiperlectura y su experiencia en el manejo de la tecnología hipertextual. En este sentido, cabe recordar que las prácticas de lectura son situadas (Barton, 1994), es decir, se originan en un contexto determinado y responden a ciertos principios y convenciones propias de una comunidad discursiva, en el caso de este trabajo, la académica.

Visto lo anterior, la lectura en internet es una actividad mediada culturalmente, donde intervienen dos sistemas simbólicos básicos: la lengua y la arquitectura hipertextual. Interesa, en esta revisión teórica, establecer el impacto del uso de medios que están construidos sobre la base de una arquitectura hipertextual, para la cognición individual: la forma de pensar y aprender de los sujetos. En este caso, el foco está en los procesos vinculados con la "gestión del conocimiento" en un contexto de alfabetización académica, que se gatillan a partir de la lectura en la red: búsqueda y selección (filtro) de información, aplicación de esta, generación del conocimiento, transmisión y divulgación del mismo (Salinas, 2005).

Dicha arquitectura hipertextual está construida sobre una cantidad enorme de enlaces de tipo multimodal (códigos verbal y no verbal), lógica de configuración que se proyecta a través de un artefacto material tecnológico de tipo informático digital que, de acuerdo a lo planteado por Monereo y Pozo (2008), es producto de la última revolución experimentada por el sistema cultural y tecnológico de los seres humanos. Una de las consecuencias más profundas de dicha revolución es la transformación cognitiva. Esta impacta directamente en los sistemas de representación, fundamentalmente los vinculados al sistema de memoria y conocimiento, es decir, aquellos que se ocupan del almacenamiento. Respecto de la tecnología informática, la creación de la internet y su estructura hipertextual, funciona como una memoria externa que, imitando al cerebro humano, da origen a una "representación mental virtual", en tanto asume, al mismo tiempo, la condición de vehículo y objeto explícito de representación (Monereo y Pozo, 2008).

En el escenario cultural actual, la transición hacia una "representación mental virtual" no ha sido tan rápida como el progreso material tecnológico que la origina y la sustenta. Entre las razones aludidas para explicar esta situación, los especialistas españoles Monereo y Pozo (2008) plantean que en la interacción con el artefacto hipertextual se ha privilegiado la dimensión pragmática, es decir, que en la última década los esfuerzos han estado orientados al manejo de esta tecnología solo como una herramienta y al desarrollo de las competencias en los sujetos a un nivel de usuarios. Esta perspectiva, válida por cierto, retardaría la incorporación de la internet como un 
objeto que merece atención en sí mismo, de acuerdo a una perspectiva epistémica, que releva la necesidad de conocer los principios, la lógica mediante las cuales opera, como un modelo que, al ser internalizado, transforma los modos de pensar y aprender.

A continuación, se aborda la "representación mental virtual" que se caracterizaría, como en toda interacción con un sistema de representación cultural, por tres procesos: implicitación, reificación e incorporación restringida (Monereo y Pozo, 2008).

a) La implicitación consiste en la internalización de las convenciones de conocer y aprender en la interacción con el objeto externo, propio del sistema de representación de una cultura. En un determinado momento, las convenciones se automatizarían y, por tanto, se aplicarían de forma inconsciente.

Este proceso se advierte en el uso del artefacto hipertextual cuando los estudiantes intentan establecer relaciones de significado entre las fuentes consultadas en la red. En un primer momento, esta actividad se realiza de forma intuitiva e ingenua, con la libertad otorgada por los enlaces electrónicos que unen los fragmentos de información. A medida que aprenden a navegar por las lexías, la búsqueda, selección y organización de la información tiene como referente criterios establecidos en la comunidad donde se originan las prácticas para conocer y aprender, en nuestro caso, la académica. Gradualmente se logra articular la forma de operar del sistema de representación digital, que enlaza el contenido con los propios objetivos de consulta y los requerimientos de producción de un discurso específico para presentar el resultado de la revisión linkográfica sobre un tema.

b) La reificación ocurre cuando un sistema cultural (y sus formas de aprender y conocer) supera la condición de representación mental y se conforma en un objeto real y universal. Por ejemplo, los modos de interacción con la realidad operan con las convenciones de internet, como se aprecia en las tareas académicas, donde se transfieren los protocolos discursivos que caracterizan a la red, la cual organiza el contenido en forma multimodal y de forma radial en diferentes direcciones, estableciendo diversas relaciones entre los fragmentos de información. Paulatinamente, se observa la lógica hipertextual a través de las estrategias utilizadas por los estudiantes para representar los caminos recorridos en la consulta de fuentes linkográficas, como lo demuestra la elaboración de un mapa mental para registrar la intertextualidad alcanzada, el cual además contiene íconos de los buscadores, imágenes representativas de las ideas seleccionadas y links a las páginas visitadas, lo que reproduce la arquitectura del hipertexto.

c) La incorporación restringida se refiere a las limitaciones que impone el propio sistema cognitivo humano en su asimilación del sistema cultural restringiéndolo, en una primera instancia, a un uso pragmático que limita su potencialidad epistémica. Este proceso se manifiesta en los sujetos cuando utilizan estrategias como el mapa de lectura, donde están aplicando una lógica hipertextual para relacionar ideas de distintas fuentes, y relegan a un segundo lugar, el aporte del artefacto tecnológico digital, confiriéndole calidad de herramienta que constituye un mero soporte de la transcripción del proceso de intertextualidad. 
Esta representación virtual, tan compleja como el sistema cognitivo al que pretende imitar, requiere en el individuo el desarrollo de ciertas competencias para "gestionar el conocimiento", cuya meta es lograr la generación del mismo. Este objetivo, en alguna medida, es cumplido por el estudiante en su rol de "internauta", quien a través de los link elegidos, expresión de la intertextualidad, tiene la posibilidad de producir sus propios textos (Escolano, 2006: 39) y convertirse en un escritor- lector, que "interpreta lo dicho por otros con anterioridad, lector que tiende puentes de intertextualidad porque toma las palabras dichas por otros como referencia a través de la cita, la paráfrasis, la traducción o la transcripción, o bien para dar una respuesta, hacer una réplica o una refutación y una discusión" (Castelló, 2007: 20).

La concepción del sujeto como un "intérprete", rol materializado precisamente mediante los saltos realizados entre uno y otro link que abastecen continuamente su sistema cognitivo, responde a un paradigma cibernético en la hermenéutica, de naturaleza autopoietica, en constante recreación y reelaboración (Iser, 2005). Desde esta perspectiva, la construcción del saber se caracteriza por ser "intersubjetiva, de los contenidos transmitidos y de los lenguajes en que se expresan, que es la vía más compatible con la intertextualidad resultante y con las formas intermodales de comunicación" (Escolano, 2006: 19).

\section{Metodología}

El estudio de caso es un método de investigación utilizado para recoger información $\mathrm{y}$, al mismo tiempo, corresponde a una experiencia de aprendizaje activa que, en esta instancia, se desarrolla con internet. Este tipo de actividad se caracteriza por enfrentar a los estudiantes a una situación- problema real y auténtica, debidamente contextualizada, para que sea resuelta de manera flexible, en forma individual o en grupo. En general, este desafío moviliza los conocimientos previos que los sujetos tienen sobre un contenido específico y complejo, e invita a buscar mayor información para poder responder a interrogantes, las cuales son formuladas por el profesor/tutor y/o generadas por los propios aprendices (Marcelo, 2002).

Considerando las características anteriores, el estudio de caso constituye la herramienta más pertinente para alcanzar el objetivo del trabajo: describir la práctica de lectura hipertextual desarrollada en un ámbito académico e identificar y analizar los procesos cognitivos implicados.

El estudio se aplicó a tres estudiantes de Magíster en Educación, dos de ellos vinculados a la profesión docente y el tercero del ámbito de la medicina. La selección de los sujetos se realizó de acuerdo a los siguientes criterios: cursar la etapa final del programa de postgrado y evidenciar manejo de los principios y lógica del hipertexto para desarrollar tareas académicas.

La tarea específica consistió en la búsqueda, selección y asociación de información sobre el tema alfabetización académica mediante la navegación en la red. El tiempo asignado para su desarrollo fue de noventa minutos. 
La recogida y registro de los datos de la lectura en internet fue realizada on line, con apoyo del software Netsupport. De esta forma, fue posible observar las acciones realizadas por los sujetos durante el desarrollo de la tarea.

En tanto, el análisis de los datos recogidos se realizó a partir de la perspectiva fenomenográfica o teoría de la variación (Marton, 1994), por cuanto el interés de este estudio es la identificación de las formas cualitativamente diferentes en que los tres sujetos de la muestra experimentan la lectura hipertextual en un contexto académico. La fenomenografía permitió identificar las variaciones de la lectura en internet, las cuales fueron descritas en función de categorías definidas a partir de la revisión teórica sobre los procesos de la "representación mental virtual" propuestos por Pozo y Monereo (2008).

A continuación, se describen la tarea desarrollada y el software empleado en el estudio de caso.

\section{Tarea de lectura hipertextual}

La tarea de lectura hipertextual consistió en una búsqueda de información en internet, similar a una "caza del tesoro" (Adell, 2003), pero con un nivel de complejidad, en cuanto a los procesos de selección y relación requeridos, acordes a un programa de postgrado en educación y al marco del proyecto de investigación de alfabetización académica hipertextual en el que se inserta. El diseño consideró los procesos cognitivos involucrados en la lectura hipertextual según los principios teóricos expuestos y un modelo pedagógico denominado "Enseñanza para la comprensión con nuevas tecnologías", desarrollado por la Escuela de graduados de la Universidad de Harvard (Stone et al., 2006).

El modelo pedagógico que subyace a la tarea ejecutada, de acuerdo a lo planteado por Stone et al. (2006), concibe la comprensión como la capacidad de tener un desempeño flexible para presentar un tema: explicarlo, justificarlo, extrapolarlo, relacionarlo y aplicarlo. El desempeño demanda a los estudiantes el desarrollo del pensamiento y la creatividad para utilizar lo aprendido y generar conocimiento. Para lograr este objetivo, el modelo abarca cuatro dimensiones, a saber: "(1) el conocimiento de conceptos importantes, (2) métodos de razonamiento e indagación disciplinarios, (3) propósitos y limitaciones de las diferentes esferas de comprensión y (4) formas de expresar la comprensión ante auditorios particulares" (Stone et al., 2006: 37). Cada una de estas dimensiones fue considerada en la tarea de lectura hipertextual, que contempló una secuencia de cuatro actividades.

a) Lectura de una síntesis general del tema a investigar. Esta actividad tiene como objetivo proporcionar un input al estudiante, que le permita apropiarse de las ideas centrales y los conceptos clave del tema a investigar. De este modo, se asegura que el alumno tenga un conocimiento previo que guíe su búsqueda de información en internet, fundamentalmente al momento de elegir los sitios y links que consulta.

La síntesis es un documento breve con una extensión máxima de una página y media, escrito en una modalidad discursiva expositiva y producido por el profesor de la 
asignatura "Leer y escribir en el siglo XXI". Se trata de una revisión global de los antecedentes más relevantes sobre el objeto de la consulta, que incluye las referencias de los autores y especialistas utilizados. Además, como punto de partida de la búsqueda en la red, se presenta un listado de sitios web que han sido seleccionados, según criterios de confiabilidad y actualidad respecto del contenido, y de accesibilidad referido a la navegación (Fainholc, 2004). Estas direcciones electrónicas estaban disponibles para los estudiantes en los marcadores del navegador de internet.

El tema de la búsqueda es "alfabetización académica" y fue definido en el marco de una asignatura que tiene por objetivo desarrollar las competencias de escritura requeridas en un programa de postgrado, con apoyo de la herramienta tecnológica hipertextual.

b) Formulación de una pregunta sobre el tema. El propósito de esta actividad es acotar el ámbito de la búsqueda a una de las ideas o uno de los conceptos clave presentado en la síntesis del tema. Se apostó por la autonomía del estudiante, quien de acuerdo a sus conocimientos e intereses, formulaba una pregunta de investigación que orientaba y monitoreaba su búsqueda en internet, especialmente la selección de información.

Se consideró el planteamiento de la pregunta como una ventaja porque formaba parte del repertorio de estrategias en el trabajo con fuentes, que los estudiantes utilizaron antes de producir ensayos. Por lo tanto, constituía un modo de indagación propio de la asignatura en la que se inscribe la tarea.

c) Búsqueda y selección de información sobre el tema en internet. La finalidad de esta actividad fue navegar por la red en busca de información para responder a la pregunta formulada en la etapa anterior. Para ello, se solicitó al estudiante usar el navegador web Internet Explorer, al que accedía a través de un ícono del escritorio del ordenador. Aun cuando existía libertad para elegir el motor de búsqueda, se sugirió al alumno utilizar el google académico y el metabuscador copernic, por su grado mayor de especialización disciplinaria en la organización del conocimiento científico publicado.

Se instruyó a los sujetos para que durante la navegación tomaran nota de la información seleccionada y de las referencias linkográficas, utilizando Word u otra herramienta de Office. Los apuntes debían contener, además, un registro de las relaciones entre las ideas recopiladas. De hecho, la intertextualidad fue uno de los requisitos de los trabajos de la asignatura, y los estudiantes estaban familiarizados con el concepto, que identificaban como una de las características centrales del hipertexto.

Adicionalmente, se entregó un apunte con el resumen de las normas APA más usadas para apoyar la elaboración de las referencias linkográficas de las fuentes desde donde se seleccionó la información.

d) Elaboración de un mapa de lectura hipertextual. El sentido de esta actividad fue representar en forma global la información seleccionada, respondiendo a la pregunta de la búsqueda y presentando gráficamente las relaciones generadas por el estudiante. 
El mapa como producto final (output) del estudio de caso planteaba una tarea académica auténtica, que supone (o debiera suponer) su difusión en la comunidad que conforman los pares y docente de la asignatura. La definición de los destinatarios condiciona dicha representación porque debía ser comprensible para otros. Para los efectos del estudio, el mapa tenía como receptores a los investigadores.

La elaboración del mapa fue asistida por alguno de los programas de Office que seleccionó el estudiante considerando su manejo de las herramientas disponibles comúnmente en un ordenador.

\section{Software Netsupport School}

El software Netsupport School fue elegido para apoyar la aplicación del estudio de caso, considerando sus características técnicas, el costo de adquisición y las herramientas que permiten múltiples ejecuciones, especialmente pensadas para el ámbito educativo.

El Netsupport School 10.50 (versión 2009) está diseñado para ser ejecutado en ordenadores autónomos y en redes informáticas. Es posible cargar el software desde un servidor de archivos en red, pero es necesario adquirir una licencia por cada estación de trabajo donde se instala.

Esta aplicación multimedia incluye dos programas: Control (Tutor) y Cliente (Estudiante).

El programa Control o tutor es instalado en las estaciones de trabajo que se utilizan para monitorear remotamente otros PCs. En tanto, el componente denominado Cliente o estudiante se instala en los equipos que son controlados remotamente. El diseño del software ofrece un interfaz amigable que facilita su manejo.

En el estudio de caso propuesto, el componente Control o Tutor fue utilizado por los investigadores para monitorear en tiempo real (cronómetro incluido) y gestionar la tarea de lectura hipertextual en el escritorio de los equipos de los estudiantes (usuarios finales). Una de las herramientas empleadas fue la barra de visualización rápida que permite ver, simultáneamente y en miniatura, todas las pantallas de los ordenadores, así como acceder a una en particular ampliando la imagen. Además, existe la posibilidad de capturar datos de la pantalla mediante fotografias o videos. En cuanto a las funciones, se destaca el modo monitorizar que admite: el acceso a las aplicaciones de office que son usadas por los sujetos y las páginas web que estos consultan; la monitorización del teclado y el mouse para observar el avance de la escritura en Word y hacer un seguimiento a la selección de links durante la navegación por sitios web.

\section{Resultados}

De acuerdo a lo descrito en la metodología, el estudio de caso se aplicó en una asignatura de Magíster en Educación, con una muestra de tres sujetos. Los aspectos en torno a los cuales se realiza el análisis de la tarea de lectura en Internet, monitoreada on line, corresponden a los procesos de representación mental virtual, a saber: 
implicitación, reificación e incorporación restringida (Monereo y Pozo, 2008) revisados en el marco teórico.

\section{Sujeto 1}

a) Implicitación: El estudiante realiza la tarea de lectura con varias ventanas abiertas que consulta una y otra vez en forma recursiva, con transiciones que van desde cinco segundos a dos minutos, dependiendo de la acción desarrollada. Los saltos más rápidos se producen entre una fuente de Internet, generalmente desde el resumen, las conclusiones y la bibliografía de un artículo, y el documento de síntesis sobre el tema a investigar, donde se verifica algún concepto o referencia de autores, como lo demuestra el movimiento del mouse sobre el archivo Word. En cuanto a las transiciones que superan el minuto, se observa una lectura atenta del fragmento de un artículo o sitio web, y la acción de copiar y pegar información de la red en la ficha (ver figura 1).

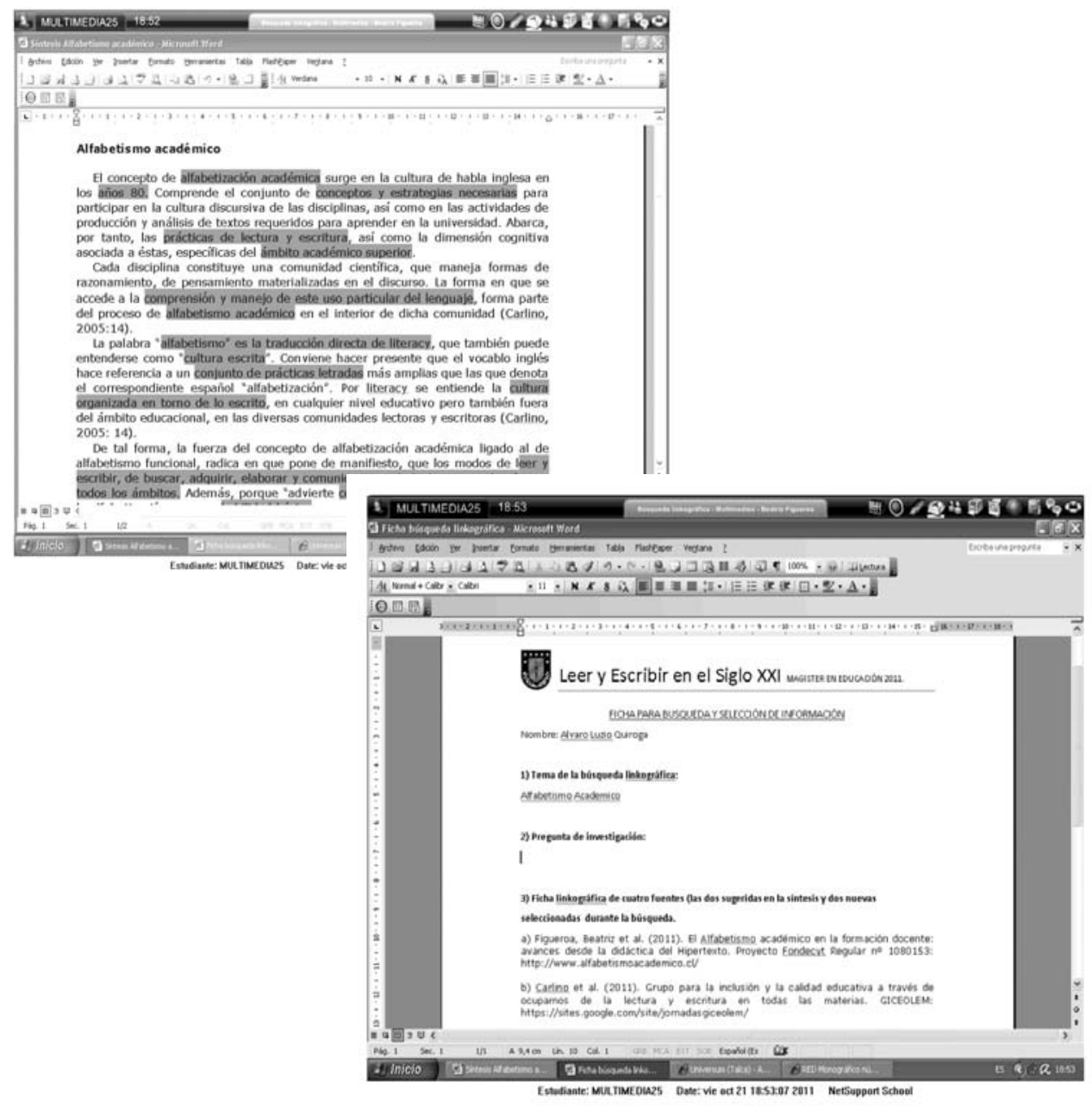

Figura 1: Transición de lectura hipertextual 
Respecto de la revisión linkográfica, esta se caracteriza por una hiperlectura direccional (Fainholc, 2004), donde los criterios que la guían obedecen a una lógica y protocolos claramente académicos, específicamente la confiabilidad y actualidad. Por lo tanto, se construye el sentido a partir de: i) la síntesis del contenido entregada por el profesor, utilizando el marcador de color de Word para destacar los conceptos principales y sus definiciones; ii) la ficha de la tarea de hiperlectura para pegar información seleccionada y la referencia linkográfica que incluye la fecha de consulta; y iii) las fuentes especializadas, artículos citados en la síntesis del profesor, como páginas web de grupos de investigación en la línea de la alfabetización académica.

b) Reificación: La búsqueda y selección de la información en las fuentes linkográficas responde a procedimientos automáticos que evidencian una lectura esquemática, que si bien está orientada por protocolos académicos (revisión de los abstract, palabras clave, introducción y referencias de los paper electrónicos o web), está fuertemente influida por las convenciones de la lectura en la red. A pesar de que esta hiperlectura se caracteriza por ser superficial, en extremo parcial y filtrada, igualmente presenta procedimientos reflexivos que permiten en un tiempo acotado y breve, la interpretación coherente de los fragmentos y enlaces consultados. Esta intertextualidad da lugar a una representación (mapa mental) en un nivel de plan o bosquejo susceptible de ser ampliado o desarrollado con mayor profundidad.

c) Incorporación restringida: Internet es utilizado, desde una perspectiva pragmática, como una herramienta de búsqueda y selección de información, que el sujeto maneja a un nivel de usuario experto. Demuestra en la navegación, facilidad para administrar simultáneamente varias ventanas, pasar con rapidez de una a otra, elegir las fuentes de consulta linkográficas y localizar información en los apartados clave de los artículos (resumen, conclusiones, bibliografía). Mantiene el foco de la hiperlectura, todas sus acciones están orientadas a responder una pregunta relacionada con un aspecto específico del tema de investigación.

En cuanto a la lógica hipertextual, el estudiante elabora un mapa mental (ver figura 2) con el programa Power Point, iniciando por el centro de la hoja, donde registra la pregunta formulada que guió su búsqueda. En torno a la interrogante, pega las referencias linkográficas completas de dos de las cuatro fuentes seleccionadas e incluye la fotografía de una investigadora, especialista en el tema, y una imagen de la interfaz del sitio web de una revista de educación, que muestra el resumen de uno de los artículos revisados. Mientras construye el mapa, despliega las ventanas de office: Formato (fuente y tamaño de la letra, colores de las mismas, etc.) y Herramientas (importar, recortar y disminuir imágenes). Sin embargo, su dominio de la arquitectura hipertextual solo le permite vincular imágenes y referentes de las fuentes con sus direcciones electrónicas, de acuerdo a protocolos superficiales del sistema. Queda de manifiesto la limitación de su potencial epistémico en la medida que no asocia ideas ni conceptos que caracteriza a la compleja red de relaciones que constituye Internet. 


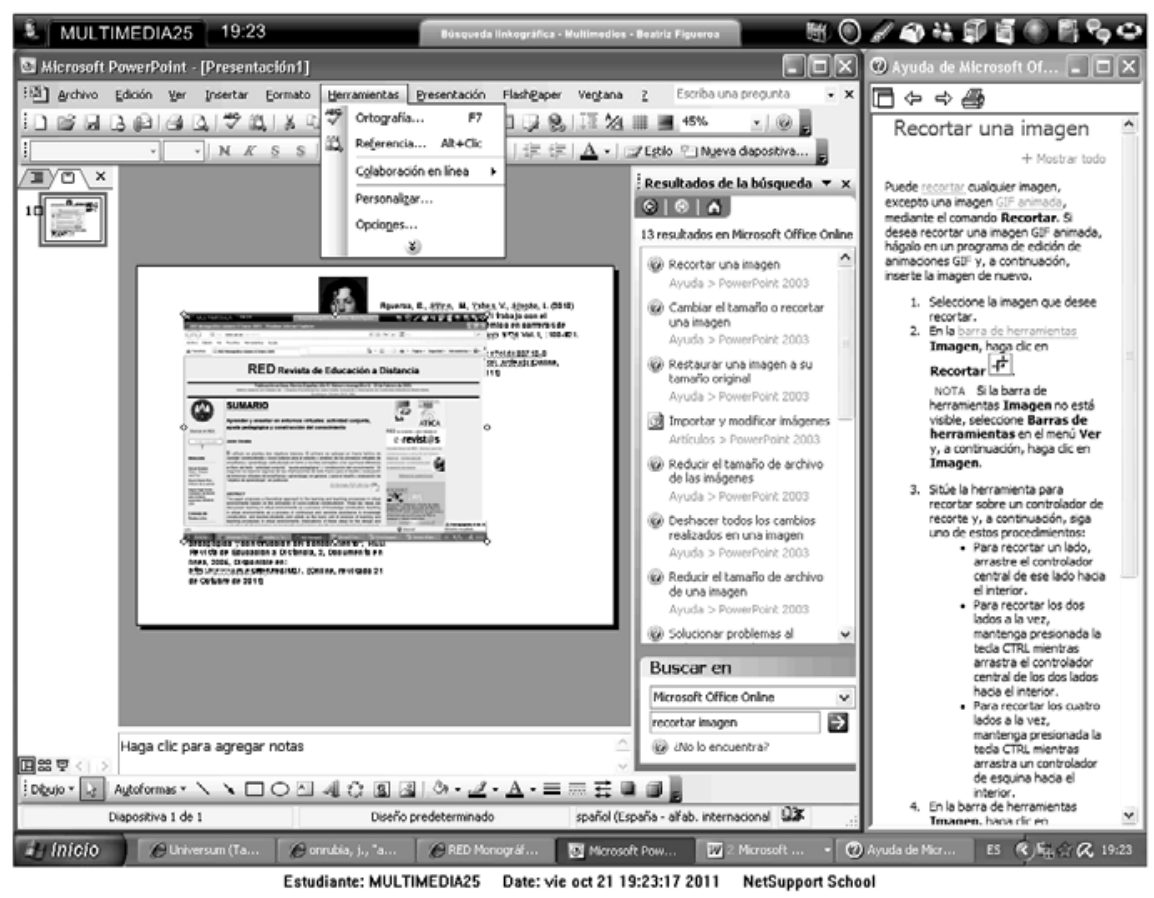

Figura 2: Construcción mapa mental

\section{Sujeto 2}

a) Implicitación: El estudiante, antes de iniciar la tarea de hiperlectura, ha invertido aproximadamente treinta minutos en la revisión de su correo Gmail y Twitter (figura 3). Cuando decide comenzar, trabaja con varias ventanas abiertas que consulta en forma secuencial (Fainholc, 2004): la síntesis del tema de investigación, Internet Explorer, y la ficha de búsqueda. Revisa rápidamente la síntesis proporcionada por el profesor, luego se dirige al navegador y escribe como palabras claves de la búsqueda "estudios alfabetismo académico". Aparece en los resultados un listado de diez enlaces. Elige el cuarto, se trata de un artículo publicado en una Revista de Humanidades, disponible en la biblioteca científica electrónica Scielo (de Chile). Revisa otros tres artículos, dos de ellos son parte de las referencias de la síntesis y uno tiene relación con el tema solo por la palabra "alfabetización" en su título. A continuación, el sujeto copia en la ficha cada una de las referencias linkográficas sin la fecha de consulta. Finaliza con la formulación de una pregunta orientada hacia la alfabetización inicial de los primeros años de escolaridad, olvidando que el tema se centra en el ámbito académico universitario. 


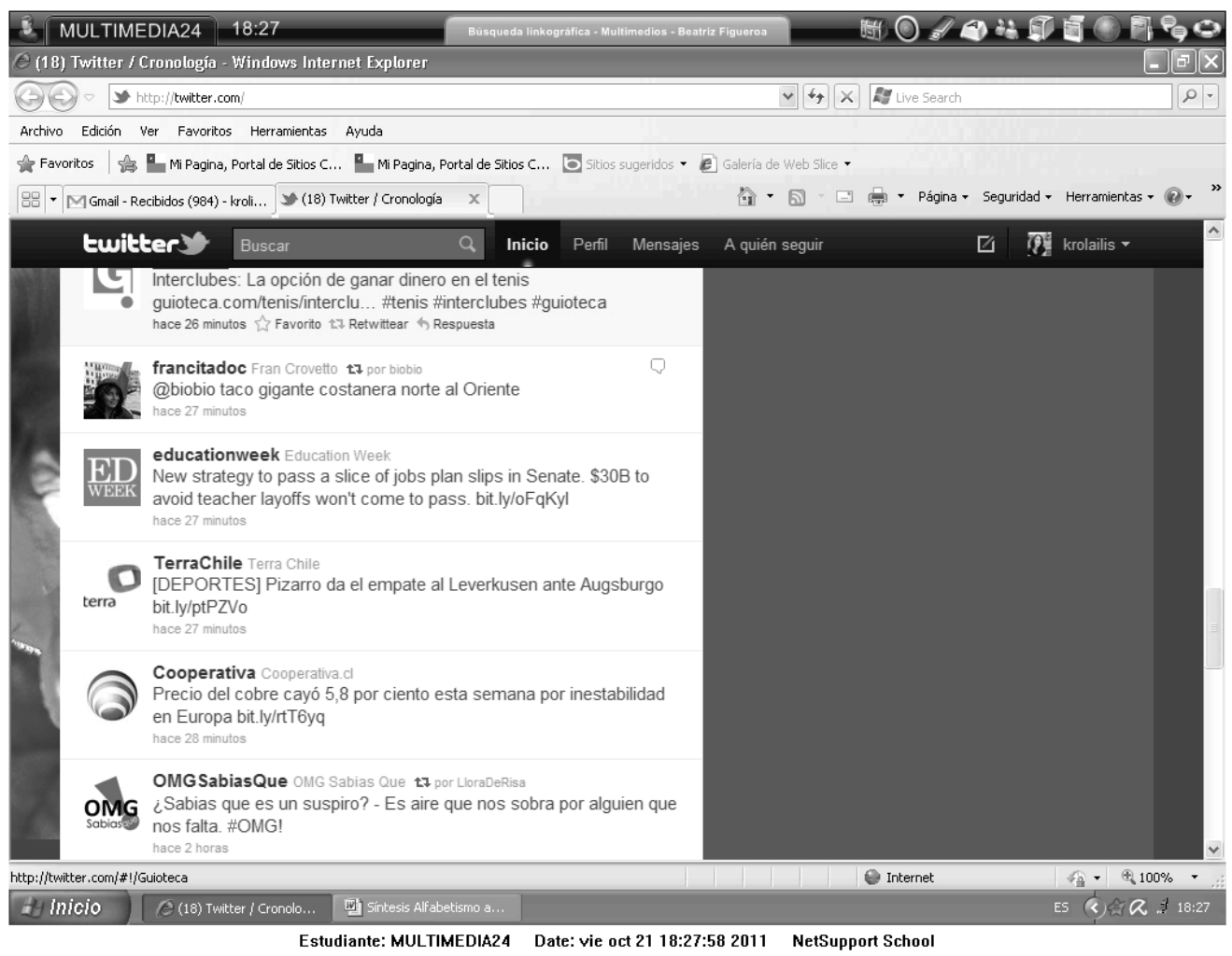

Figura 3: Uso de internet para fines recreativos

b) Reificación: La búsqueda y selección de la información en las fuentes linkográficas responde a una navegación básica que intenta ceñirse a los protocolos y convenciones de la red, pero como hiperlectura secuencial (salto de un enlace a otro en forma sucesiva), con predominio de la lógica de una lectura lineal tradicional, el sujeto se enfrenta a una fragmentación de la información proveniente de varias fuentes. La consecuencia en la construcción de sentido es la dificultad para seleccionar y relacionar en forma coherente la información y enlaces revisados. Esto se evidencia en la incapacidad del sujeto para elaborar una representación gráfica (el mapa mental) del resultado de su tarea de búsqueda linkográfica, alcanzando a registrar únicamente su pregunta de investigación, que no responde al tema propuesto, y una imagen de un libro aludiendo a la lectura tradicional (figura 4). 


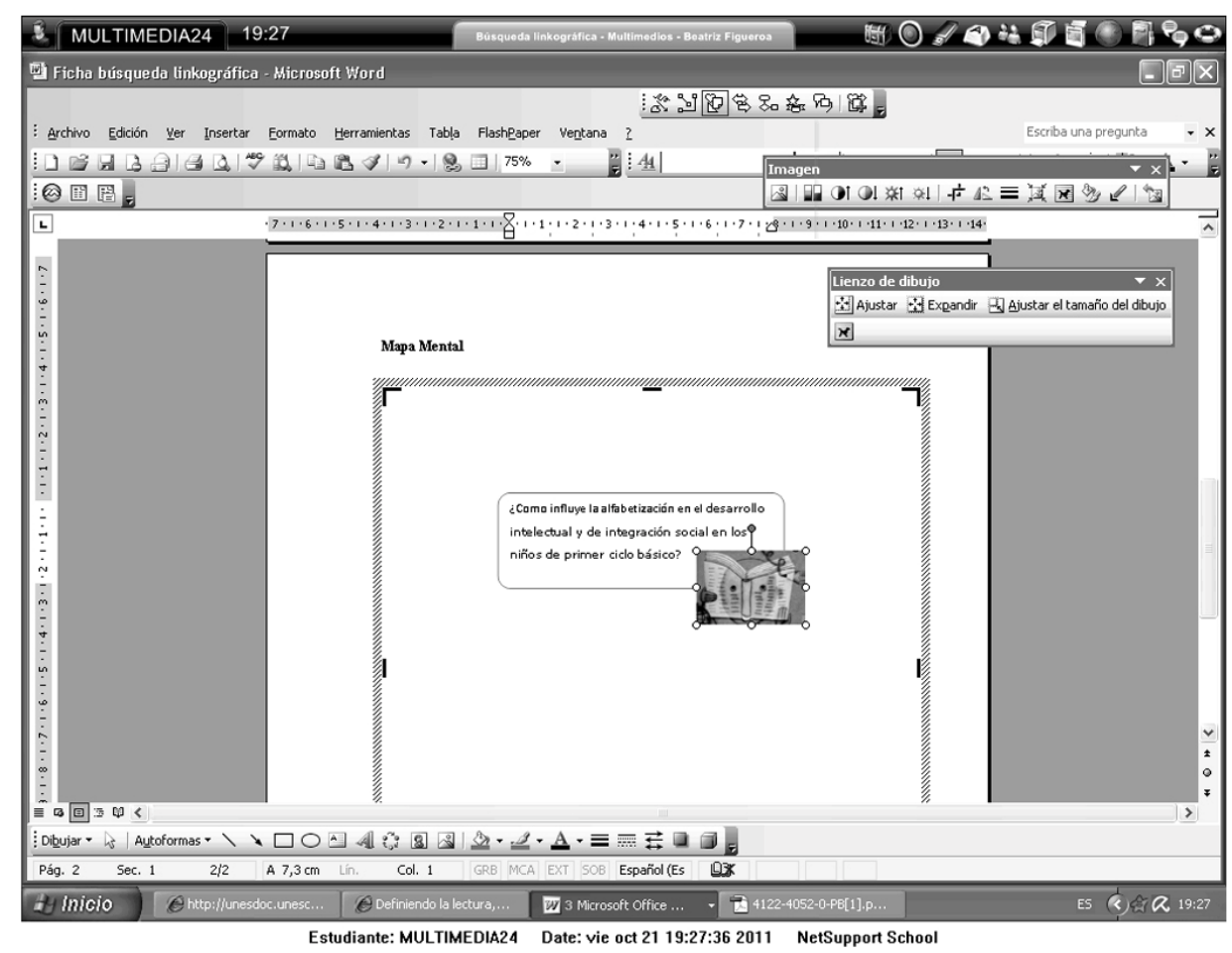

Figura 4: Construcción deficiente de mapa mental

c) Incorporación restringida. La red es considerada, desde una perspectiva pragmática, como una herramienta de contacto e interacción, atendiendo a la media hora que el sujeto invirtió en Gmail y Twitter antes de comenzar la tarea de hiperlectura. En la búsqueda y selección de información, el estudiante es un usuario inexperto, demostrando su manejo limitado de: i. la lógica de la no linealidad y recursividad que caracteriza al soporte hipertextual y posibilita la relación de la información e ideas de las fuentes consultadas (intertextualidad); ii. la navegación con propósitos académicos que demanda la definición del tema, la formulación de preguntas, y el registro de conceptos e ideas clave durante el proceso. Además de la escasa internalización de las convenciones de la comunidad de postgrado de la cual forma parte para seleccionar las fuentes.

\section{Sujeto 3}

a) Implicitación: El estudiante comienza la tarea abriendo el archivo Word con la síntesis del tema elaborada por el profesor. Diez minutos después de leer el documento, revisa la ficha de búsqueda linkográfica y anota el tema. Elige el navegador Internet Explorer y escribe las palabras claves sobre el tópico. Su lectura en la red es lineal, consulta los artículos en orden, empleando entre 10 y 15 minutos en cada uno, y pasa con atención por las páginas donde se encuentran la introducción y el marco teórico. A 
continuación, el sujeto copia en la ficha cuatro referencias de los artículos estudiados, con la dirección electrónica, omitiendo la fecha de consulta, y toma apuntes enunciando ideas sobre el tema de investigación, pero no las desarrolla (ver figura 5). Finaliza con la formulación de una pregunta, que parafrasea el título de uno de los artículos seleccionados.

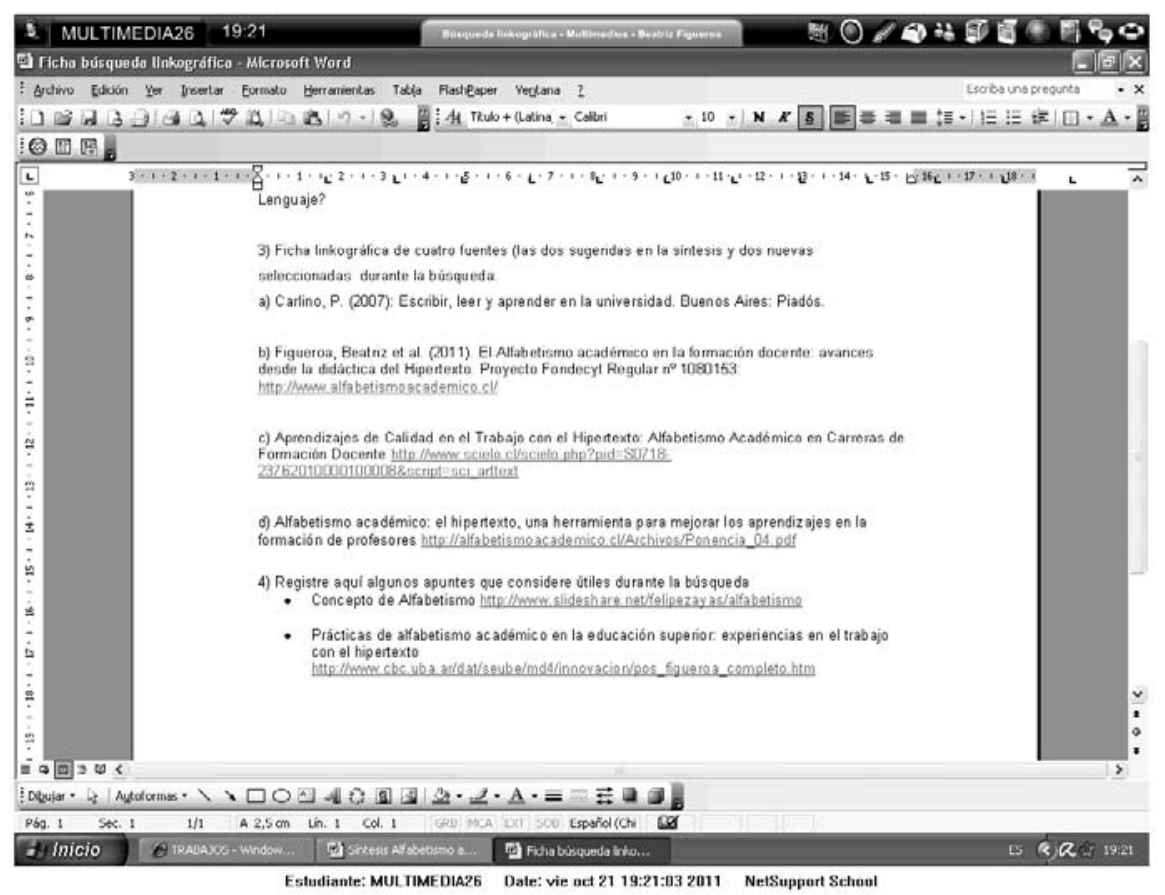

Figura 5: Toma de apuntes

b) Reificación: La búsqueda y selección de información en las fuentes linkográficas, en el caso de este sujeto, consiste en una lectura lineal, centrada en la revisión teórica para extraer la definición del tema. Esto permite dar sentido y coherencia a la fragmentación de la información de Internet. La única evidencia del resultado de la navegación es la ficha, por cierto incompleta, dado que el estudiante no tuvo tiempo de elaborar el mapa mental con la representación de la tarea ejecutada.

c) Incorporación restringida. La ficha, referente del proceso de hiperlectura, revela que la habilidad para asociar la información seleccionada de las fuentes consultadas está reducida a la identificación de similitudes, considerando que se privilegió la definición del tema. La estrategia empleada frente a la fragmentación de Internet prueba el escaso o nulo dominio de la arquitectura hipertextual, que mediante enlaces interrelaciona en forma compleja una serie de textos, requiriendo de la interpretación del sujeto para dar coherencia. 
A partir de los resultados presentados, desde la perspectiva fenomenográfica, el sujeto 1 abre la variación porque en la tarea de hiperlectura proporciona evidencias que reflejan su tránsito hacia la compleja "representación mental virtual", con mayor desarrollo en los procesos de implicitación, reificación e incorporación restringida (Monereo y Pozo, 2008). La calidad demostrada por el estudiante en los mencionados procesos se relaciona directamente con el manejo de la arquitectura hipertextual y las convenciones para navegar entre un enlace y otro, en forma no lineal y recursiva.

En cambio, los sujetos 2 y 3 en su interacción con Internet presentan, por lo menos en dos de las categorías, aspectos comunes (invariantes). De acuerdo a la fenomenografía, ellos demuestran un desarrollo incipiente en los procesos descritos, principalmente debido a la lógica de la lectura lineal y secuencial que aplican en la configuración hipertextual.

\section{Conclusiones}

Las evidencias empíricas obtenidas en este estudio de caso sobre lectura hipertextual, realizado con apoyo del soporte informático digital, tienen, a juicio de las autoras, doble mérito. Esto debido a que las tareas propuestas y el apoyo de la tecnología no solo constituyen procedimientos valiosos para la recogida de datos, sino que, también, son instancias de aprendizaje significativas, tanto para los estudiantes como para los investigadores. Visto lo anterior, es necesario continuar y avanzar en esta línea. Por esto, aspectos a considerar en el diseño y ejecución de este tipo de estudio de caso son: la selección de una herramienta software para monitorear la tarea de lectura hipertextual que realizan los estudiantes, monitorear en modalidad sincrónica su desarrollo y registrar los productos de su interacción con la red.

El producto de esta aplicación, en lo inmediato, contribuye a describir los procesos cognitivos de la lectura en internet, cuestión que ha sido escasamente abordada por la investigación empírica. Dicha descripción permite observar la relevancia no solo de la comprensión lectora, sino del manejo de las prácticas no lineales de hiperlectura que exigen los nuevos soportes de la información. En el caso del sujeto 2, su poca habilidad como hiperlector, aun cuando muestra un manejo de internet a nivel recreativo (al interactuar, por ejemplo, con Twitter), lo lleva a desarrollar de manera deficiente las tareas encomendadas. En el caso del sujeto 3, se evidencia que es un lector lineal, tradicional, lo que entorpece su avance en la hiperlectura, aun cuando puede llevar a cabo las tareas de manera más o menos adecuada. El sujeto 1, a diferencia de sus compañeros, demuestra ser un buen hiperlector, ya que es capaz de interactuar con diversas fuentes y de realizar adecuadamente las tareas propuestas. Finalmente, es notorio que existen grandes diferencias entre los procesos cognitivos que llevan a cabo los sujetos y que estos inciden en la calidad del producto final de las tareas.

En el mediano plazo, los resultados obtenidos aportan a la elaboración, implementación y validación de un diseño didáctico que potencie la alfabetización académica hipertextual en programas de postgrado. Dicho diseño apunta a las exigencias propias de los estudios en ese nivel, que incluyen una capacidad de búsqueda, selección y comprensión de la información crítica, que se plasme en la 
escritura de textos académicos. Para lograr cumplir con estas exigencias, es preciso que los estudiantes tengan conciencia de sus prácticas de lectura y, en particular, de cómo enfrentar y desarrollar prácticas de hiperlectura.

Adicionalmente, los hallazgos de la investigación permiten establecer criterios más específicos para elegir un software que considere el desarrollo de competencias de lectura y escritura académicas, y el aprendizaje de la lógica hipertextual, siguiendo patrones de los procesos cognitivos de los sujetos.

El uso de un software implica, además, hacerse cargo de la alfabetización digital, que sirve de soporte a la red. En este sentido, conviene insistir en que los estudiantes manejen el ordenador e internet y aprendan a navegar, internalizando la lógica hipertextual. Como afirman Monereo y Pozo (2008), esto ocurrirá si se asume, desde una perspectiva epistémica, la arquitectura de internet como objeto de estudio y no solo como una simple herramienta. Para avanzar en esta dirección, se podría tomar como modelo a los expertos que realizan una búsqueda estratégica, aquellos que son capaces de gestionar el conocimiento. En esta línea y considerando la tecnología disponible actualmente, surgen interrogantes para abordar en futuras investigaciones respecto de los soportes utilizados por los usuarios expertos y su beneficio en el desarrollo de la hiperlectura: ¿qué dispositivos digitales prefieren dichos usuarios para acceder a la red?, ¿cuáles son las características de los artefactos elegidos que favorecen la consulta y el tratamiento de la información con propósitos académicos?, ¿cómo los aparatos empleados condicionan los procesos de representación mental virtual de los hiperlectores?

\section{Referencias bibliográficas}

ADELL, J. (2003). Internet en el aula: a la caza del tesoro. EDUTEC, Revista Electrónica de Tecnología Educativa, 16. [En línea]. Disponible en http://edutec.rediris.es/Revelec2/revelec16/adell.htm. [Consulta: 10/06/2011]

ALBARELLO, (2010) Leer/navegar en Internet. Un estudio comparativo entre jóvenes escolarizados y docentes porteños sobre las formas de lectura en la computadora. Tesis Doctoral. Universidad Austral, Buenos Aires, Argentina. [En línea]. Disponible en http://es.scribd.com/doc/185813385/Tesis-doctoral-deFrancisco-Albarello-Leer-Nagevar-en-Internet. [Consulta: 20/07/2014]

BARTON, D. (1994). Literacy. Oxford: Blackwell.

CARLINO, P. (2007). Escribir, leer y aprender en la universidad. $3^{\text {era }}$ edic. Buenos Aires: Fondo de Cultura Económico.

CASTELLÓ, M. (Coord.) (2007). Escribir y comunicarse en contextos científicos y académicos. Conocimientos y estrategias. Barcelona: Graó.

COLL, C., MONEREO, C. (Eds.). (2008). Psicología de la educación virtual. Madrid: Morata. 
COLOM, A. (2002). La (de) construcción del conocimiento pedagógico. Barcelona: Paidós.

CHARTIER, A. (2004). Enseñar a leer y escribir. Una aproximación histórica. México: Fondo de Cultura Económica.

DESCRIPTORES DE DUBLÍN (2005). Shared «Dublin» descriptors for the Bachelor's, Master's and Doctoral awards. (Draft 1.31 working document on JQI meeting in Dublin. 2004PC). [En línea]. Disponible en http://tempus.ac.rs/here/tl_files/Dokumenti/Dublinski\%20deskriptori.pdf [Consulta: 16/02/2014]

ESCOLANO, B. (Ed.) (2006). Currículum editado y sociedad del conocimiento. Texto, multimedialidad y cultura de la escuela. Valencia: Tirant Lo Blanche.

FAINHOLC, B. (2004). Lectura crítica en Internet: análisis y utilización de recursos tecnológicos en educación. Rosario: Homo Sapiens.

FIGUEROA, B., AILlON, M., YÁÑEZ, V., AJAGÁN, L. (2010). Aprendizajes de calidad en el trabajo con el hipertexto: Alfabetismo académico en carreras de formación docente. Revista Universum, 25 (1), 100- 121.

ISER, W. (2005). Rutas de la interpretación. México: Fondo de Cultura Económica.

MARCELO, C. (2002). Estrategias didácticas para un aprendizaje activo en teleformación. En C. Marcelo, D. Puente, M. A. Ballesteros, A. Palazón ELearning teleformación. Diseño, desarrollo y evaluación de la formación a través de Internet, 81- 100. Barcelona: Gestión.

MARTON, F. (1994). Phenomenography. In Husen \& Postlethwaite (Vol 8 Eds.). International Encyclopedia of Education (2nd ed.), 4424-4429. New York: Pergamon.

MAYER, R. Y MORENO, R. (2003). Nine ways to reduce cognitive load in multimedia learning. Educational Psychologist, 38 (1), 43-52.

MONEREO, C., FUENTES, M., SÀNCHEZ, S. (2000). Internet Search and Navigation Strategies Used by Experts and Beginners. Interactive Educational Multimedia, 1, 24-34.

MONEREO, C., POZO, J. (2008). El alumno en entornos virtuales: Condición, perfil y competencias, en Coll, C. y Monereo, C. (eds.) Psicología de la educación virtual, 109- 131. Madrid: Morata.

PARODI, G. (ed.) (2010). Alfabetización académica y profesional en el siglo XXI: Leer y escribir desde las disciplinas. Santiago de Chile: Planeta.

PISCITELLI, A. (2009). Nativos digitales. Dieta Cognitiva, inteligencia colectiva y arquitecturas de la participación. Buenos Aires: Santillana.

RUEDA, R. (2007). Para una pedagogía del hipertexto. Una teoría de deconstrucción y la complejidad. Barcelona: Anthropos. 
SALINAS, J. (2005). La gestión de los Entornos Virtuales de Formación. Universidad de las Islas Baleares. Grupo de Tecnología Educativa. En el Seminario Internacional: La calidad de la formación en red en el Espacio Europeo de Educación Superior. [En línea]. Disponible en http:/gte.uib.es/pape/gte/sites/gte.uib.es.pape.gte/files/gestioEVEA_0.pdf.

[Consulta: 13/06/2011]

SEVILLANO, M.L. Y VÁZQUEZ, E. (2014). Análisis de la funcionalidad didáctica de las tabletas digitales en el espacio europeo de educación superior. RUSC. Universities and Knowledge Society Journal, 11(3), 67-81. doi http://doi.dx.org/10.7238/rusc.v11i3.1808

STONE, M., RENNEBOHM, K. Y BREIT, L. (2006). Enseñar para la comprensión con nuevas tecnologías. $2^{\text {da }}$ edic. Buenos Aires: Paidós.

WARSCHAUER, M. (2000). The death of cyberspace and the rebirth of CALL. English Teachers' Journal 53, 61-67. [En línea]. Disponible en http://www.gse.uci.edu/person/warschauer_m/cyberspace.html. [Consulta: 20/06/2011]

\section{Correspondencia con los autores}

Mariana AILLON NEUMANN

Facultad de Educación, Universidad de Concepción

Edmundo Larenas 335, Barrio Universitario

Concepción, Chile

e-mail: maillon@udec.cl

Beatriz FIGUEROA SANDOVAL

Facultad de Educación, Universidad de Concepción

Edmundo Larenas 335, Barrio Universitario

Concepción, Chile

e-mail: bfiguero@udec.cl

\section{Angie NEIRA MARTÍNEZ}

Departamento de Español, Universidad de Concepción

Edmundo Larenas s/n, Barrio Universitario

Concepción, Chile

e- mail: angieneira@udec.cl 